\title{
Valor adaptativo e habilidade competitiva de azevém resistente e suscetível ao iodosulfuron em competição com o trigo
}

\author{
Franciele Mariani(1), Leandro Vargas ${ }^{(2)}$, Dirceu Agostinetto(3), Marcos André Nohatto(4), \\ Ana Claudia Langaro ${ }^{(3)}$ e Thiago Vieira Duarte ${ }^{(3)}$
}

\begin{abstract}
(1)Instituto Federal de Educação, Ciência e Tecnologia do Rio Grande do Sul, Campus Sertão, Rodovia RS 135, Km 25, Distrito Engenheiro Luiz Englert, CEP 99170-000 Sertão, RS, Brasil. E-mail: marianifranciele@gmail.com (2)Embrapa Trigo, Rodovia BR-285, Km 294, Caixa Postal 3081, CEP 99050-970 Passo Fundo, RS, Brasil. E-mail: leandro.vargas@embrapa.br ${ }^{(3)}$ Universidade Federal de Pelotas, Campus Universitário, s/no, Caixa Postal 354, CEP 96160-000 Capão do Leão, RS, Brasil. E-mail: agostinetto@ig.com.br, namelia.langaro@gmail.com, duartevieira.thiago@gmail.com ${ }^{(4)}$ Instituto Federal Catarinense, Campus Avançado Sombrio, Avenida Prefeito Francisco Lummertz Júnior, oo 818, Januária, CEP 88960-000 Sombrio, SC, Brasil. E-mail: marcosnohatto@hotmail.com
\end{abstract}

Resumo - O objetivo deste trabalho foi identificar e comparar valores adaptativos dos biótipos de azevém (Lolium multiflorum) resistente e suscetível ao herbicida iodosulfuron e avaliar sua habilidade competitiva com o trigo (Triticum aestivum). Para avaliar o valor adaptativo, os biótipos foram semeados em vasos e coletados a cada 10 dias após a emergência, até os 80 dias. Em cada coleta, avaliaram-se estatura, área foliar e massa seca da parte aérea, e, a partir destas avaliações, foram calculadas variáveis indiretas para comparar o crescimento entre os biótipos. A habilidade competitiva foi determinada em experimento de série de substituição, tendo-se variado a proporção da cultura e do competidor. As proporções testadas foram: 100:0 (estande puro do trigo), 75:25, 50:50, 25:75 e 0:100\% (estande puro do biótipo de azevém resistente ou suscetível); já as variáveis avaliadas foram área foliar e massa seca. Os resultados dos estudos comparativos de crescimento mostraram similaridade entre os biótipos de azevém resistente e suscetível ao iodosulfuron, o que não caracterizou efeito pleiotrópico decorrente da resistência. Quanto à habilidade competitiva, a competição interespecífica é prejudicial para o biótipo resistente, enquanto, para o biótipo suscetível, independe o competidor. A cultura do trigo mostra habilidade competitiva superior a dos biótipos de azevém resistente e suscetível.

Termos para indexação: Lolium multiflorum, Triticum aestivum, competição interespecífica, herbicida, planta daninha.

\section{Adaptive value and competitive ability of ryegrass resistant and susceptible to iodosulfuron in competition with wheat}

\begin{abstract}
The objective of this work was to identify and compare adaptive values of ryegrass (Lolium multiflorum) biotypes resistant and susceptible to the herbicide iodosulfuron and to evaluate their competitive ability with wheat (Triticum aestivum). To evaluate the adaptive value, the biotypes were sown in pots and collected every 10 days after emergence, up to 80 days. At each collection, height, leaf area, and dry matter of shoots were assessed, and, from these evaluations, indirect variables were calculated to compare the growth between the biotypes. The competitive ability was determined in a replacement series experiment, varying the proportion of the culture and of the competitor. The proportions tested were: 100:0 (wheat in pure stand), $75: 25,50: 50,25: 75$, and $0: 100 \%$ (resistant or susceptible ryegrass biotype under pure stand); the evaluated variables were leaf area and dry matter. The results of the comparative studies of growth showed similarity between ryegrass biotypes resistant and susceptible to iodosulfuron, which does not characterize pleitropic effect due to resistance. Regarding competitive ability, interspecific competition is harmful to the resistant biotype, whereas, for the susceptible biotype, there is no effect of the competitor. The wheat crop shows greater competitive ability than resistant and susceptible ryegrass biotypes.
\end{abstract}

Index terms: Lolium multiflorum, Triticum aestivum, interspecific weed competition, herbicide, weed.

\section{Introdução}

A competição de plantas daninhas com culturas tem sido considerada a principal causa biótica que gera perdas de produtividade (Délye et al., 2013a), tendo chegado a $34 \%$, em média, em todo o mundo (Oerke, 2006). Para minimizar as perdas, tem-se utilizado intensivamente e de forma inadequada o controle químico, o que agravou o problema devido à seleção de plantas daninhas resistentes aos herbicidas.

Pesq. agropec. bras., Brasília, v.51, n.6, p.710-719, jun. 2016

DOI: $10.1590 / \mathrm{S} 0100-204 X 2016000600002$ 
A resistência aos inibidores da enzima acetolactato sintase (ALS) tem evoluído rapidamente, confirmada pelo grande número de espécies registradas como resistentes (Heap, 2014). Entre elas, destaca-se o azevém (Lolium multiflorum Lam.), principal competidor na cultura do trigo (Triticum aestivum L.) e identificado como resistente ao herbicida iodosulfuron nas principais regiões produtoras da região Sul do Brasil (Heap, 2014).

Com a evolução de populações de difícil controle, o valor adaptativo de alelos resistentes pode ser acompanhado da existência de custos de adaptação, e estes efeitos pleiotrópicos podem ser negativos ou positivos em dado ambiente, dependendo do alelo relacionado (Vila-Aiub et al., 2009b; Délye et al., 2013b). Um exemplo de alteração no valor adaptativo pode ser observado na espécie Lolium rigidum Gaudin, com mecanismo de resistência causado pelo aumento do metabolismo mediado por P450, o qual apresenta reduzidas taxa de crescimento relativo (TCR) e taxa de assimilação líquida (TAL), bem como menor habilidade de competição (Vila-Aiub et al., 2009a). Outro exemplo são os biótipos de azevém suscetíveis ao glifosato, os quais apresentam maior capacidade competitiva que os biótipos resistentes (Vargas et al., 2005; Ferreira et al., 2008). Desse modo, as plantas se adaptam às mudanças e modificam a alocação dos recursos (Vila-Aiub et al., 2009b).

O impacto da mutação que causa resistência aos inibidores da enzimaALS depende do ponto da mutação e da espécie envolvida, o que pode conferir maior efeito no crescimento de plantas e competitividade (Tardif et al., 2006) ou, então, não representar nenhum efeito (Yu et al., 2010; Li et al., 2013).

Para analisar as características dos biótipos, são utilizados estudos de crescimento que detectam possíveis diferenças funcionais e estruturais entre eles (Christoffoleti, 2001). Já os experimentos em série de substituição são utilizados para estudar a competição inter e intraespecífica (Cousens, 1991). Neste tipo de experimento, a população total é mantida constante e a proporção entre as duas espécies é variável, o que torna possível comparar as produtividades das associações com a do monocultivo e indicar qual genótipo ou espécie é mais competitivo (Cousens, 1991).

A caracterização de biótipos resistentes e suscetíveis aos inibidores da enzima ALS é importante para a compreensão do seu comportamento no ambiente, para avaliar a sua capacidade de competição com a cultura do trigo e para propor práticas de manejo que reduzam a evolução da resistência.

O objetivo deste trabalho foi identificar e comparar valores adaptativos de biótipos de azevém resistente e suscetível ao herbicida iodosulfuron e avaliar sua habilidade competitiva com o trigo.

\section{Material e Métodos}

Os experimentos foram conduzidos em casa de vegetação, pertencente à Faculdade de Agronomia Eliseu Maciel, da Universidade Federal de Pelotas, no Município de Capão do Leão, RS (3152'00"S, $\left.52^{\circ} 21^{\prime} 24^{\prime \prime} \mathrm{W}\right)$. Os tratamentos foram dispostos em delineamento experimental inteiramente casualizado, com quatro repetições para cada um, e foram conduzidos entre agosto e novembro de 2011.

No primeiro experimento, cada unidade experimental foi composta por vasos com capacidade para $8 \mathrm{~L}$ e diâmetro de $23 \mathrm{~cm}$, preenchidos com solo classificado como Argissolo Vermelho-Amarelo (Santos et al., 2013), contendo três plantas distribuídas de forma equidistante. A correção da fertilidade do solo foi realizada seguindo a análise química.

Os tratamentos consistiram de plantas de biótipos de azevém resistente (Joi) e suscetível ao herbicida iodosulfuron e de oito épocas de coleta: 10, 20, 30, 40, 50, 60, 70 e 80 dias após a emergência (DAE). Os biótipos resistentes foram oriundos do Município de Jóia (28 $\left.38^{\prime} 51^{\prime \prime S}, 54^{\circ} 07^{\prime \prime} 21^{\prime \prime W}\right)$ e os suscetíveis, de Pelotas ( $\left.31^{\circ} 48^{\prime} 04^{\prime \prime} \mathrm{S}, 52^{\circ} 24^{\prime} 50^{\prime \prime} \mathrm{W}\right)$.

As variáveis avaliadas, em cada coleta, foram: estatura de plantas, com auxílio de régua milimetrada, da base da planta até o ápice da última folha desenvolvida; área foliar, com medidor de área foliar, modelo LI-3100C (LI-COR Biosciences, Lincoln, NE, EUA); e massa seca da parte aérea (MSPA), mensurada pela secagem do material vegetal em estufa, a $60^{\circ} \mathrm{C}$, até atingir massa constante.

Foram calculadas as seguintes variáveis indiretas, de acordo com Magalhães (1979): taxa de crescimento absoluto (TCA), em grama por dia, usada para medir a velocidade média de crescimento ao longo do período de observação, por meio da equação: $\mathrm{TCA}=\left(\mathrm{MSPA}_{2}-\mathrm{MSPA}_{1}\right) /\left(\mathrm{T}_{1}-\mathrm{T}_{2}\right)$, em que $\mathrm{MSPA}_{1} \mathrm{e}$ $\mathrm{MSPA}_{2}$ são a variação da MSPA em duas amostras consecutivas tomadas nos tempos $T_{1}$ e $T_{2}$; taxa de 
crescimento foliar relativo (TCFR), em $\mathrm{g} \mathrm{g}^{-1}$ por dia, a qual representa o aumento de área foliar em determinado período de tempo, com uso da equação: TCFR $=\left(\operatorname{LnAF}_{2}-\operatorname{LnAF}_{1}\right) /\left(T_{2}-T_{1}\right)$, em que Ln é o logaritmo neperiano, e $\mathrm{AF}_{1}$ e $\mathrm{AF}_{2}$ representam a área foliar nos tempos $T_{1}$ e $T_{2}$; razão de área foliar (RAF), em $\mathrm{cm}^{2} \mathrm{~g}^{-1}$, que expressa a área foliar útil para a fotossíntese, obtida pela equação: $\mathrm{RAF}=\left(\mathrm{AF}_{1}+\mathrm{AF}_{2}\right) /$ $\left(\mathrm{MSPA}_{1}+\mathrm{MSPA}_{2}\right)$; e taxa assimilatória líquida (TAL), em $\mathrm{g} \mathrm{cm}^{-2}$ por dia, a qual representa a capacidade da planta em aumentar sua MSPA em função de sua superfície assimilatória, em determinado período de tempo, calculada por meio da equação: $T A L=\left(M_{S P A}-\right.$ $\left.\mathrm{MSPA}_{1}\right)\left(\mathrm{LnAF}_{2}-\mathrm{LnAF}_{1}\right) /\left(\mathrm{AF}_{2}-\mathrm{AF}_{1}\right)\left(\mathrm{T}_{2}-\mathrm{T}_{1}\right)$.

Os dados obtidos foram analisados quanto à normalidade, pelo teste de Shapiro-Wilk, e à homocedasticidade; posteriormente, foram submetidos à análise de variância, a 5\% de probabilidade. Quando houve significância estatística, realizou-se análise de regressão para o fator épocas de avaliação. Para as variáveis estatura, área foliar, MSPA, TCA e RAF, os dados foram ajustados à equação exponencial: $\mathrm{y}=\mathrm{ae}^{\mathrm{bx}}$, em que y é a variável resposta de interesse; $\mathrm{x}$ é $\mathrm{o}$ número de dias acumulados; e é a função exponencial; e a e b são os parâmetros estimados da equação, em que a é a diferença entre os pontos máximo e mínimo da variável, e b é a declividade da curva.

Os dados da variável TCFR foram ajustados à seguinte equação de regressão sigmoidal logística: $\mathrm{y}=\mathrm{a} /\left[1+\left(\mathrm{x} / \mathrm{x}_{0}\right) \mathrm{b}\right]$, em que y representa a taxa de crescimento; $x$ são os dias após a emergência; e a, $\mathrm{x}_{0}$ e b são os parâmetros da equação, sendo que a é a diferença entre os pontos máximo e mínimo da curva, $\mathrm{x}_{0}$ são os dias que proporcionam $50 \%$ de resposta da variável e b é a declividade da curva.

Para avaliar a habilidade competitiva entre azevém e trigo, foi realizado estudo composto por dois experimentos conduzidos em vasos com capacidade volumétrica para $4 \mathrm{~L}$ e diâmetro da superfície de $17,5 \mathrm{~cm}$. O tipo de solo e a correção foram idênticos aos descritos anteriormente. $\mathrm{O}$ experimento inicial foi realizado para determinar a população de plantas por metro quadrado a partir da qual a MSPA por unidade de área torna-se independente da população, de acordo com a "lei de produção final constante" (Radosevich et al., 2007). Foram testadas as populações de 1, 2, 4, 8, $16,32,64$ e 128 plantas por vaso (equivalentes a 42,83 , $167,333,667,1.333,2.667$ e 5.333 plantas por metro quadrado), pertencentes à cultivar de trigo Fundacep Horizonte e ao azevém suscetível e resistente.

A variável MSPA foi quantificada aos 60 DAE pela pesagem da parte aérea das plantas após serem secas em estufa com circulação forçada de ar, a $60^{\circ} \mathrm{C}$, por 72 horas. Para a análise dos dados, utilizou-se a produção recíproca para determinar a população de plantas em que a MSPA torna-se constante. De acordo com os resultados, a população de plantas em que a MSPA tornou-se independente da população, na média das espécies, foi a de 1.992 plantas por metro quadrado, equivalente a 48 plantas por vaso.

A partir dos dados do monocultivo, foi realizado o experimento para determinar a habilidade competitiva dos biótipos de azevém com o trigo. Os tratamentos consistiram de proporções dos biótipos de azevém suscetível e resistente e da cultivar de trigo Fundacep Horizonte. As proporções testadas foram: 100:0 (estande puro do trigo), 75:25, 50:50, 25:75 e 0:100\% (estande puro do biótipo de azevém resistente ou suscetível).

As variáveis avaliadas foram área foliar e MSPA aos 60 DAE, como descrito anteriormente. Para a análise dos dados, foi utilizado o método da análise gráfica da produtividade relativa (PR) (Cousens, 1991). Quando a PR resulta em: linha reta, a habilidade da espécie ou do biótipo em interferir sobre a outra é equivalente; linha côncava, ocorre prejuízo no crescimento de um ou de ambos os competidores; e linha convexa, há benefício no crescimento de um ou de ambos os competidores. Já quando a produtividade relativa total (PRT) é: igual a um (linha reta), ocorre competição pelo(s) mesmo(s) recurso(s); superior a um (linha convexa), não há competição, uma vez que o suprimento de recursos supera a demanda ou as espécies apresentam diferentes demandas pelo(s) recurso(s) do meio; e inferior a um (linha côncava), há antagonismo, com prejuízo mútuo ao crescimento de ambas as espécies (Cousens, 1991).

Os índices de competitividade relativa (CR), os coeficientes de agrupamento relativo $(\mathrm{K})$ e os de competitividade (C) foram calculados na proporção de $50 \%$ dos competidores. Neste caso, CR representa o crescimento comparativo do biótipo (azevém resistente ou suscetível) em relação ao competidor (trigo); K indica a dominância relativa de uma espécie ou biótipo sobre a outra; e $\mathrm{C}$ aponta qual espécie ou biótipo manifesta-se mais competitiva (Cousens, 1991). Os índices $\mathrm{CR}, \mathrm{K}$ e $\mathrm{C}$ foram considerados diferentes 
quando, em pelo menos duas proporções, ocorreram significâncias pelo teste $t$ (Bianchi et al., 2006).

Para a análise estatística da PR, primeiro, foram calculadas as diferenças para os valores de PR obtidos nas proporções de 25, 50 e 75\% de plantas, em relação aos valores pertencentes às retas hipotéticas que foram obtidas nas respectivas proporções. O teste t, a $5 \%$ de probabilidade, foi utilizado para testar as diferenças nos índices estimados em relação à reta hipotética (Passini et al., 2003).

Os resultados obtidos para área foliar e MSPA, expressos em valores médios por planta, foram submetidos à análise de variância. Quando o teste $\mathrm{F}$ indicou significância a 5\% de probabilidade, as médias dos tratamentos foram comparadas pelo teste de Dunnett, a 5\% de probabilidade, tendo-se considerado as respectivas monoculturas como testemunhas.

\section{Resultados e Discussão}

Houve interação entre os fatores biótipo e época de avaliação para todas as variáveis, exceto para TAL, em que o fator biótipo apresentou efeito principal. A estatura e a área foliar dos biótipos aumentaram exponencialmente com o avanço das épocas de avaliação (Figura 1 AeB). Ao se considerar o coeficiente exponencial das equações, o comportamento entre os biótipos foi similar. Em relação ao acúmulo da MSPA, o comportamento entre os biótipos também foi semelhante, tendo diferido apenas aos 70 e 80 DAE (Figura $1 \mathrm{C}$ ). Indivíduos com maior acúmulo de massa seca em menor espaço de tempo mostram maior habilidade competitiva, quando em competição (Radosevich et al., 2007). Assim, quanto ao acúmulo de MSPA, a habilidade competitiva entre os biótipos foi similar.

A relação entre os biótipos resistente e suscetível foi semelhante para a TCA (Figura 1 D). Além disso, ao se considerar como mais competitivo o biótipo que apresenta TCA inicial mais alta, não houve diferença entre os biótipos no início do desenvolvimento. Biótipos de Bidens pilosa L. resistentes aos inibidores da enzima ALS apresentaram TCA inicial menor que a do biótipo suscetível; porém, igualaram-se a este a partir dos 49 dias (Christoffoleti, 2001). Conforme esses autores, esses resultados explicam por que o biótipo resistente é mantido em baixa frequência na ausência do herbicida como agente de pressão de seleção.

A TCFR foi semelhante para ambos os biótipos ao longo das avaliações, tendo diminuído a produtividade diária de matéria por grama de planta, após os $40 \mathrm{DAE}$, pois, à medida que os biótipos se desenvolvem, diminuem sua capacidade fotossintética, o que aumenta as perdas por respiração (Figura $2 \mathrm{~A}$ ). A RAF declinou com o avanço das avaliações (Figura 2 B), tendo diferido apenas aos 20 DAE. Esse resultado pode decorrer do autossombreamento, do surgimento de tecidos e estruturas não assimilatórias, bem como do secamento e da queda de folhas com a idade da planta (Urchei et al., 2000).

De acordo com as variáveis avaliadas, podese inferir que os biótipos resistente e suscetível apresentam poucas diferenças adaptativas, que provavelmente podem ser atribuídas ao fato de o local de origem não ser o mesmo. Resultados semelhantes foram observados entre biótipos resistentes e suscetíveis aos inibidores da enzima ALS, quanto às características de desenvolvimento das espécies Bidens subalternans DC. (Lamego et al., 2011), Cyperus difformis L. (Dal Magro, 2011), B. pilosa (Christoffoleti, 2001) e Raphanus raphanistrum L. (Li et al., 2013). Em biótipos de azevém suscetível e com resistência de nível baixo ao fluazifop, também foi verificado valor adaptativo similar entre biótipos (Fraga et al., 2013). Para biótipos de Fimbristylis miliacea (L.) Vahl resistente e suscetível a inibidores da enzima ALS, houve diferenças quanto aos seus valores adaptativos no início do florescimento; contudo, não foram relatadas diferenças em estádios iniciais de desenvolvimento (Schaedler et al., 2013). A ausência de efeitos negativos no crescimento de plantas com diferentes mutações na enzima ALS contribui para explicar a alta frequência de alelos de resistência na população (Yu et al., 2010; Li et al., 2013).

A modificação causada na enzima ALS, que confere resistência, não está correlacionada ao decréscimo de produtividade da planta (Christoffoleti, 2001). Portanto, segundo esses autores, estratégias preventivas de resistência devem ser adotadas, pois, uma vez estabelecida a população resistente, naturalmente ela não retorna para a frequência original de suscetibilidade. Cuidados maiores devem ser tomados quando as espécies de plantas daninhas 
apresentam polinização cruzada, já que pode ocorrer cruzamento entre os biótipos resistentes e suscetíveis, o que aumenta rapidamente a frequência do biótipo resistente (Powles \& Yu, 2010).

Em relação à habilidade competitiva, constatouse, de forma geral, que a cultura do trigo foi mais competitiva que ambos os biótipos de azevém (Figura 3). Houve benefício para a cultura e prejuízo para a planta daninha, o que mostra que a competição ocorreu pelos mesmos recursos do meio e que a cultura foi mais eficiente em utilizá-los. Apesar disso, como os desvios da produtividade relativa (PR) para o biótipo suscetível não foram significativos, para ambas as variáveis (Tabela 1), sua habilidade competitiva foi equivalente à do trigo. Entretanto, não foram observadas grandes diferenças no valor adaptativo entre biótipos para explicar esse resultado. Em estudo em que o trigo competiu com azevém suscetível e com resistência de nível baixo ao herbicida fluazifop, também verificou-se que a habilidade competitiva do trigo foi superior à do biótipo com resistência de nível baixo mas equivalente à do biótipo suscetível (Fraga et al., 2013).

Foram observados desvios de PR para a cultura de trigo, quanto à MSPA, nas três proporções, na competição com azevém suscetível, quando comparado à respectiva reta hipotética (Tabela 1). Já em relação ao biótipo resistente, os desvios foram positivos para a
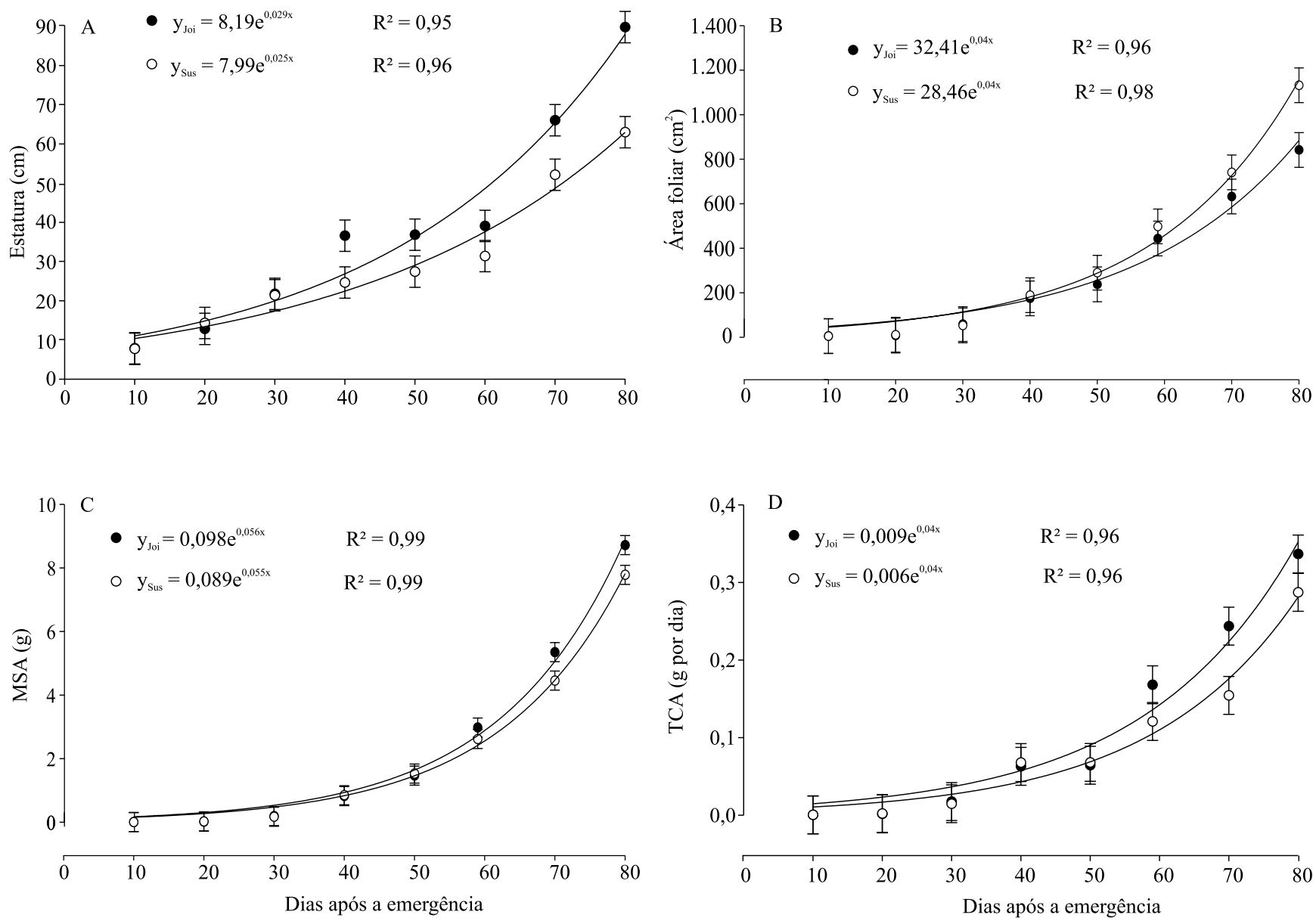

Figura 1. Estatura, área foliar, massa seca da parte aérea (MSPA) e taxa de crescimento absoluto (TCA) de biótipos de azevém (Lolium multiflorum) resistente (Joi) e suscetível (Sus) ao herbicida iodosulfuron, avaliadas dos 10 aos 80 dias após a emergência. Os pontos representam os valores médios das repetições entre biótipos, e as barras, os respectivos intervalos de confiança da média. 
cultura e negativos para o azevém, o que confirma que a cultura foi mais competitiva.

Ao analisar as diferenças para ambas as variáveis, entre as misturas, não houve diferença para a cultura ao competir com o biótipo suscetível; no entanto, houve incremento, em geral, quando a cultura participava com igual ou menor proporção de plantas na mistura com o biótipo resistente (Tabela 2). Assim, em 50\% das comparações, houve diferença para a cultura que competia com ambos os biótipos, com incremento de MSPA e área foliar; nesses casos, a cultura prefere a competição com a planta daninha à competição intraespecífica. Contudo, em 50\% das comparações, não foram observadas diferenças significativas. Fraga et al. (2013), ao avaliar azevém em competição com trigo, encontraram resultados semelhantes, em que a cultura preferiu dividir o nicho com outra espécie a competir com plantas da mesma espécie.

Para a maioria das comparações do biótipo suscetível em competição com a cultura de trigo, não houve diferença em relação ao monocultivo, ou seja, o tipo de competição foi indiferente (Tabela 2). O biótipo resistente diminuiu a MSPA e a área foliar na maioria das comparações em relação ao monocultivo, o que mostra que a competição interespecífica é desvantajosa. Quanto às misturas, ambos os biótipos diminuíram a área foliar e a MSPA quando competiram
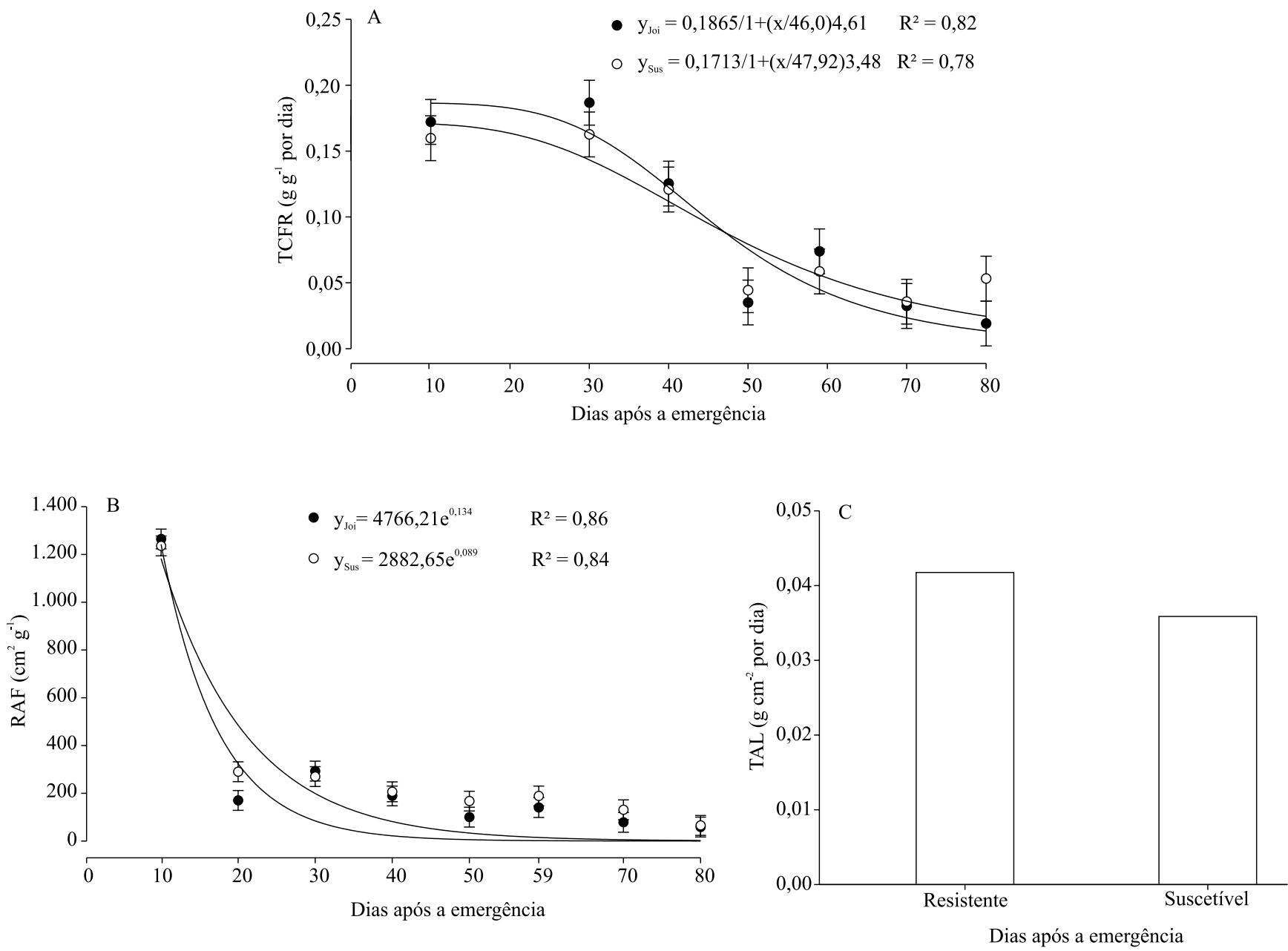

Figura 2. Taxa de crescimento foliar relativo (TCFR), razão de área foliar (RAF) e taxa assimilatória líquida (TAL) de biótipos de azevém (Lolium multiflorum) resistente (Joi) e suscetível (Sus) ao herbicida iodosulfuron, avaliadas dos 10 aos 80 dias após a emergência. Os pontos representam os valores médios das repetições entre biótipos, e as barras, os respectivos intervalos de confiança da média. 
em igual proporção à cultura, com exceção do biótipo resistente para a última variável.

Observou-se que, para ambas as variáveis, o trigo foi o mais competitivo em relação aos biótipos de azevém (Tabela 3). Em trabalho que avaliou a habilidade competitiva do trigo, este foi superior ao azevém, porém, inferior ao nabo ( $R$. raphanistrum) em proporções iguais, sendo que ambas as plantas daninhas ocupam o mesmo nicho ecológico do trigo (Rigoli et al., 2008).

Não foram relatadas diferenças na capacidade competitiva de biótipos resistentes aos inibidores da
ALS, para várias espécies (Sibony \& Rubin, 2003; Lamego et al., 2011; Légère et al., 2013). A falta de efeitos negativos em biótipos resistentes aos inibidores da enzima ALS favorece a evolução, a propagação e a competitividade dessas espécies nos sistemas agrícolas (Légère et al., 2013). Além disso, a evolução de biótipos resistentes sem custo de adaptação constitui-se como ameaça aos sistemas de cultivo, principalmente na cultura do trigo, para a qual o azevém é o principal competidor.

Efeitos negativos na morfologia e na anatomia vegetal têm sido descritos para Amaranthus powellii
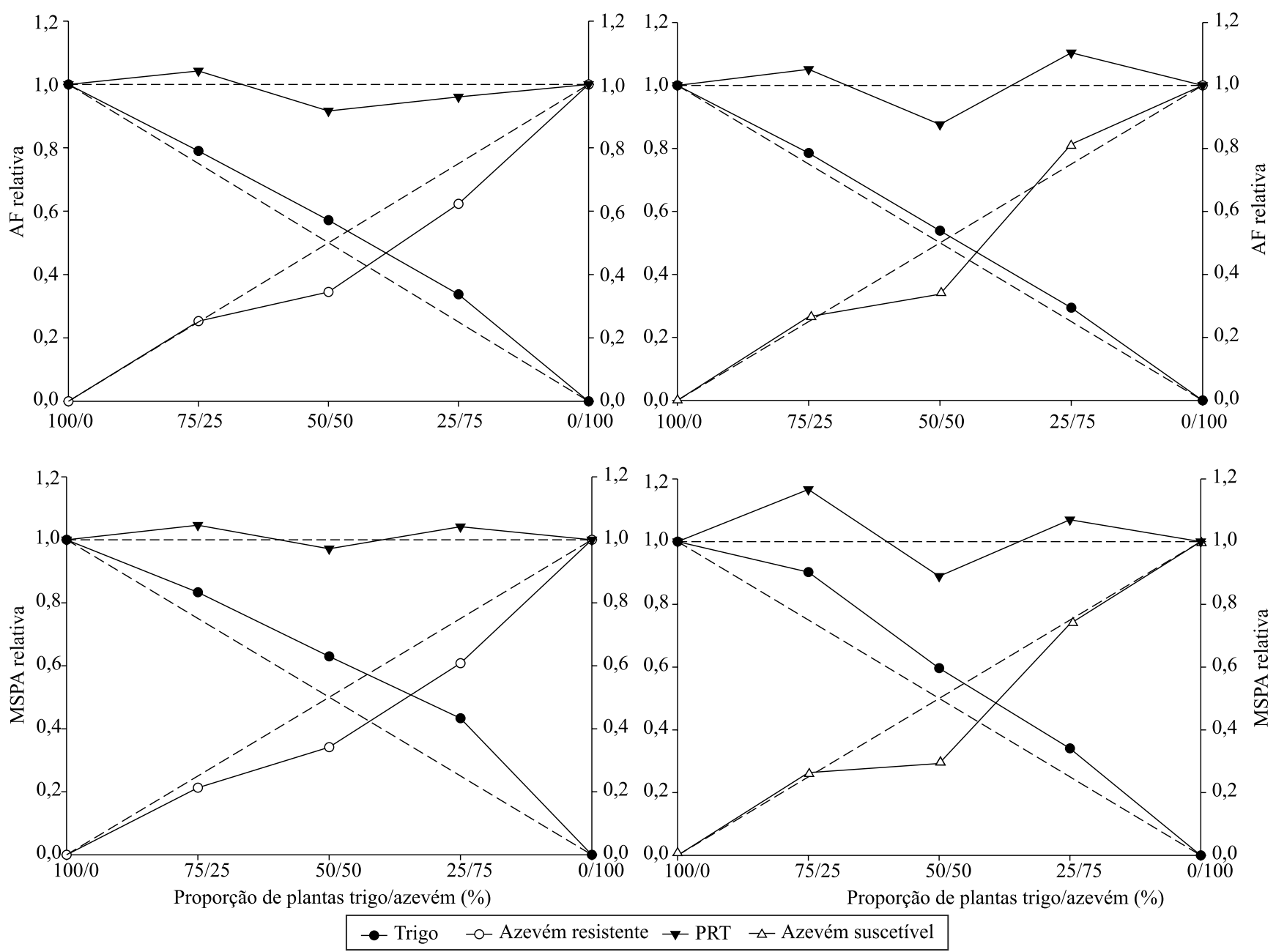

Figura 3. Produtividade relativa (PR) e total (PRT) para área foliar (AF) e massa seca da parte aérea (MSPA) de biótipos de azevém (Lolium multiflorum) resistente e suscetível ao herbicida iodosulfuron, em competição com trigo (Triticum aestivum) 'Fundacep Horizonte'. Círculos e triângulos vazios representam a PR do biótipo resistente ou suscetível; círculos cheios indicam a PR do trigo; e triângulos cheios representam a PRT. Linhas tracejadas referem-se às produtividades relativas hipotéticas, quando não há interferência de uma espécie sobre a outra. 
S.Watson resistente à enzima ALS (Tardif et al., 2006). Para biótipos de Solanum ptychanthum Dunal resistentes aos inibidores da enzima ALS, ocorreu custo adaptativo devido à mutação em condições ótimas de crescimento, apesar de apresentarem maiores germinação e crescimento vegetativo que biótipos suscetíveis em temperaturas baixas (Ashigh \& Tardif, 2011). Isso evidencia que o custo adaptativo também depende das condições ambientais às quais as plantas são submetidas. Para chegar a conclusões seguras quanto ao custo de adaptação de biótipos, é necessário trabalhar com biótipos oriundos de diferentes regiões geográficas, com a mesma alteração na enzima e com seus respectivos controles locais - biótipo suscetível (Légère et al., 2013) -, ou seja, com múltiplas populações (Cousens et al., 1997). Portanto, faz-se necessário realizar novos estudos com maior número de biótipos, com controle genético e caracterização molecular, para assegurar-se dos resultados.

Quanto à maior habilidade do trigo em competir com azevém, esse resultado não pode ser tomado como base para inferir que não há prejuízo para a cultura,

Tabela 1. Diferenças relativas de produtividade para as variáveis área foliar, massa seca da parte aérea (MSPA) e produtividade relativa total (PRT), nas proporções de plantas 75:25, 50:50 e 25:75 de biótipos de azevém (Lolium multiflorum) suscetível e resistente ao herbicida iodosulfuron, em competição com trigo (Triticum aestivum) 'Fundacep Horizonte'.

\begin{tabular}{lccc}
\hline Variável & \multicolumn{3}{c}{ Proporção de plantas associadas } \\
\cline { 2 - 4 } & \multicolumn{3}{c}{$50: 50$} \\
\hline & Área foliar $\left(\mathrm{cm}^{2}\right)$ \\
PRatrigo & $0,04 \pm 0,02^{\mathrm{ns}(1)}$ & $0,07 \pm 0,00^{*}$ & $0,09 \pm 0,01^{*}$ \\
PRbresistente & $0,00 \pm 0,00^{\mathrm{ns}}$ & $-0,16 \pm 0,01^{*}$ & $-0,13 \pm 0,02^{*}$ \\
PRT & $1,05 \pm 0,02^{\mathrm{ns}}$ & $0,92 \pm 0,01^{*}$ & $0,96 \pm 0,03^{\mathrm{ns}}$ \\
PRatrigo & $0,04 \pm 0,06^{\mathrm{ns}}$ & $0,04 \pm 0,03^{\mathrm{ns}}$ & $0,04 \pm 0,01^{*}$ \\
PRbsuscetível & $0,02 \pm 0,02^{\mathrm{ns}}$ & $-0,16 \pm 0,01^{*}$ & $0,05 \pm 0,03^{\mathrm{ns}}$ \\
PRT & $1,05 \pm 0,05^{\mathrm{ns}}$ & $0,88 \pm 0,03^{*}$ & $1,09 \pm 0,02^{*}$ \\
\hline & & $\mathrm{MSPA}(\mathrm{g})$ & \\
PRatrigo & $0,08 \pm 0,03^{\mathrm{ns}}$ & $0,13 \pm 0,02^{*}$ & $0,18 \pm 0,06^{*}$ \\
PRbresistente & $-0,04 \pm 0,02^{\mathrm{ns}}$ & $-0,16 \pm 0,02^{*}$ & $-0,14 \pm 0,02^{*}$ \\
PRT & $1,05 \pm 0,02^{\mathrm{ns}}$ & $0,97 \pm 0,02^{\mathrm{ns}}$ & $1,04 \pm 0,08^{*}$ \\
PRatrigo & $0,15 \pm 0,05^{*}$ & $0,10 \pm 0,02^{*}$ & $0,09 \pm 0,01^{*}$ \\
PRbsuscetível & $0,01 \pm 0,02^{\mathrm{ns}}$ & $-0,21 \pm 0,01^{*}$ & $0,02 \pm 0,03^{\mathrm{ns}}$ \\
PRT & $1,17 \pm 0,05^{\mathrm{ns}}$ & $0,89 \pm 0,03^{*}$ & $1,11 \pm 0,04^{*}$ \\
\hline
\end{tabular}

${ }^{(1)}$ Média \pm erro-padrão da média. *Diferença significativa pelo teste t, a $5 \%$ de probabilidade. ${ }^{\text {ns }}$ Não significativo. já que o experimento foi conduzido até os 60 DAE. Além disso, em condições de campo, as cultivares de trigo podem ter diferente capacidade de competição, e a população de plantas daninhas é variável. Ressaltase, porém, que cultivares de trigo reduziram em 80 a $85 \%$ a produtividade, ao competir com azevém e nabo, respectivamente, quando em convivência durante todo o ciclo da cultura (Lamego et al., 2013).

Tabela 2. Resposta de biótipos de azevém (Lolium multiflorum) suscetível e resistente ao herbicida iodosulfuron, em competição com trigo (Triticum aestivum) 'Fundacep Horizonte', nas diferentes proporções de plantas avaliadas, quanto à área foliar e à massa seca da parte aérea (MSPA) ${ }^{(1)}$.

\begin{tabular}{lccccc}
\hline Biótipo & \multicolumn{5}{c}{ Proporção de plantas } \\
\cline { 2 - 6 } & $100: 0$ & $75: 25$ & $50: 50$ & $25: 75$ & $0: 100$ \\
\hline \multirow{5}{*}{ Trigo } & 26,20 & $27,61^{\mathrm{ns}} \mathrm{c}$ & $29,95^{*} \mathrm{~b}$ & $35,38^{*} \mathrm{a}$ & - \\
Resistente & - & $52,41^{\mathrm{ns}} \mathrm{a}$ & $35,76^{*} \mathrm{c}$ & $43,07 * \mathrm{~b}$ & 51,85 \\
\hline Trigo & 26,20 & $27,43^{\mathrm{ns}} \mathrm{a}$ & $28,42^{\text {ns }} \mathrm{a}$ & $30,85^{\text {ns }} \mathrm{a}$ & - \\
Suscetível & - & $55,28^{\mathrm{ns}} \mathrm{a}$ & $35,07^{*} \mathrm{~b}$ & $53,08^{\text {ns }} \mathrm{a}$ & 52,00 \\
\hline & & & MSPA $(\mathrm{g})$ \\
Trigo & 0,34 & $0,37^{\mathrm{ns}} \mathrm{b}$ & $0,42^{\mathrm{ns}} \mathrm{ab}$ & $0,57^{*} \mathrm{a}$ & - \\
Resistente & - & $0,37^{*} \mathrm{a}$ & $0,30 * \mathrm{a}$ & $0,36^{*} \mathrm{a}$ & 0,44 \\
\hline Trigo & 0,34 & $0,40^{*} \mathrm{a}$ & $0,40 * \mathrm{a}$ & $0,45^{*} \mathrm{a}$ & - \\
Suscetível & - & $0,38^{\mathrm{ns}} \mathrm{a}$ & $0,21 * \mathrm{~b}$ & $0,37^{\mathrm{ns}} \mathrm{a}$ & 0,37 \\
\hline
\end{tabular}

${ }^{(1)}$ Médias seguidas de letras iguais, minúsculas nas linhas, em proporções com mistura de plantas, não diferem pelo teste de Duncan, a 5\% de probabi-

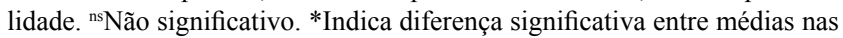
linhas, respectivamente, pelo teste de Dunnett, a 5\% de probabilidade, em relação à testemunha (monocultura de trigo).

Tabela 3. Índices de competitividade entre trigo (Triticum aestivum) 'Fundacep Horizonte' e azevém (Lolium multiflorum) suscetível e resistente ao herbicida iodosulfuron, expressos por competitividade relativa (CR), coeficientes de agrupamentos relativos (K) e de competitividade (C), aos 60 dias após a emergência.

\begin{tabular}{lcccc}
\hline Variável & $\mathrm{CR}$ & $\mathrm{K}_{\mathrm{t}}$ (trigo) & $\mathrm{K}_{\mathrm{r}}$ (azevém) & $\mathrm{C}$ \\
\hline \multicolumn{5}{c}{ Trigo x azevém resistente } \\
MSPA $^{(1)}$ & $1,87 \pm 0,14^{*(2)}$ & $1,72 \pm 0,13^{*}$ & $0,52 \pm 0,04$ & $0,29 \pm 0,03^{*}$ \\
Área foliar $\left(\mathrm{cm}^{2}\right)$ & $1,66 \pm 0,06^{*}$ & $1,33 \pm 0,01^{*}$ & $0,52 \pm 0,02$ & $0,23 \pm 0,01^{*}$ \\
\hline \multicolumn{5}{c}{ Trigo x azevém suscetível } \\
MSPA & $2,04 \pm 0,09^{*}$ & $1,50 \pm 0,15^{*}$ & $0,41 \pm 0,02$ & $0,30 \pm 0,02^{*}$ \\
Área foliar $\left(\mathrm{cm}^{2}\right)$ & $1,60 \pm 0,06^{\mathrm{ns}}$ & $1,18 \pm 0,11^{*}$ & $0,51 \pm 0,02$ & $0,20 \pm 0,02^{*}$ \\
\hline
\end{tabular}

${ }^{(1)}$ MSPA, massa seca da parte aérea. ${ }^{(2)}$ Média \pm erro-padrão da média. $K_{t}$, $\mathrm{K}_{\mathrm{s}}$ e $\mathrm{K}_{\mathrm{r}}$ são os coeficientes de agrupamentos relativos do trigo (Triticum aestivum) com o azevém (Lolium multiflorum) suscetível e resistente,

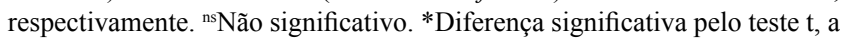
$5 \%$ de probabilidade. 


\section{Conclusões}

1. O valor adaptativo de biótipos de azevém (Lolium multiflorum) resistente e suscetível ao herbicida iodosulfuron é similar.

2. A cultura do trigo (Triticum aestivum) apresenta maior habilidade competitiva do que os biótipos de azevém resistente e suscetível ao iodosulfuron.

3. A competição interespecífica para o biótipo de azevém resistente é prejudicial, já, para o biótipo suscetível, independe o competidor.

\section{Agradecimentos}

À Coordenação de Aperfeiçoamento de Pessoal de Nível Superior (Capes), pela concessão de bolsa.

\section{Referências}

ASHIGH, J.; TARDIF, F.J. Water and temperature stress impact fitness of acetohydroxyacid synthase-inhibiting herbicide-resistant populations of eastern black nightshade (Solanum ptychanthum). Weed Science, v.59, p.341-348, 2011. DOI: 10.1614/ WS-D-10-00126.1.

BIANCHI, M.A.; FLECK, N.G.; LAMEGO, F.P. Proporção entre plantas de soja e plantas competidoras e as relações de interferência mútua. Ciência Rural, v.36, p.1380-1387, 2006. DOI: 10.1590/ S0103-84782006000500006.

CHRISTOFFOLETI, P.J. Análise comparativa do crescimento de biótipos de picão-preto (Bidens pilosa) resistente e suscetível aos herbicidas inibidores da ALS. Planta Daninha, v.19, p.75-83, 2001. DOI: $10.1590 / \mathrm{S} 0100-83582001000100009$.

COUSENS, R. Aspects of the design and interpretation of competition (interference) experiments. Weed Technology, v.5, p.664-673, 1991.

COUSENS, R.D.; GILL, G.S.; SPEIJERS, E.J. Comment: number of sample populations required to determine the effects of herbicide resistance on plant growth and fitness. Weed Research, v.37, p.1-4, 1997. DOI: 10.1111/j.1365-3180.1997.tb01816.x.

DAL MAGRO, T.; SCHAEDLER, C.E.; FONTANA, L.C.; AGOSTINETTO, D.; VARGAS, L. Habilidade competitiva entre biótipos de Cyperus difformis L. resistente ou suscetível a herbicidas inibidores de ALS e destes com arroz irrigado. Bragantia, v.70, p.294-301, 2011.

DÉLYE, C.; JASIENIUK, M.; LE CORRE, V. Deciphering the evolution of herbicide resistance in weeds. Trends in Genetics, v.29, p.1-10, 2013a. DOI: 10.1016/j.tig.2013.06.001.

DÉLYE, C.; MENCHARI, Y.; MICHEL, M.; CADET, E.; LE CORRE, V. A new insight into arable weed adaptive evolution: mutations endowing herbicide resistance also affect germination dynamics and seedling emergence. Annals of Botany, v.111, p.681-691, 2013b. DOI: 10.1093/aob/mct018.
FERREIRA, E.A.; CONCENÇO, G.; SILVA, A.A.; REIS, M.R.; VARGAS, L.; VIANA, R.G.; GUIMARÃES, A.A.; GALON, L. Potencial competitivo de biótipos de azevém (Lolium multiflorum). Planta Daninha, v.26, p.261-269, 2008. DOI: 10.1590/ S0100-83582008000200002.

FRAGA, D.S.; AGOSTINETTO, D.; VARGAS, L.; NOHATTO, M.A.; THÜRMER, L.; HOLZ, M.T. Adaptive value of ryegrass biotypes with low-level resistance and susceptible to the herbicide fluazifop and competitive ability with the wheat culture. Planta Daninha, v.31, p.875-885, 2013. DOI: 10.1590/ S0100-83582013000400014.

HEAP, I. The International Survey of Herbicide Resistant Weeds. Disponível em: $<$ http://www.weedscience.org $>$. Acesso em: 13 mar 2014.

LAMEGO, F.P.; RUCHEL, Q.; KASPARY, T.E.; GALLON, M.; BASSO, C.J.; SANTI, A.L. Habilidade competitiva de cultivares de trigo com plantas daninhas. Planta Daninha, v.31, p.521-531, 2013. DOI: $10.1590 / \mathrm{S} 0100-83582013000300004$.

LAMEGO, F.P.; VIDAL, R.A.; BURGOS, N.R. Competitiveness of ALS inhibitors resistant and susceptible biotypes of greater beggarticks (Bidens subalternans). Planta Daninha, v.29, p.457-464, 2011. DOI: 10.1590/S0100-83582011000200024.

LÉGÈRE, A.; STEVENSON, F.C.; BECKIE, H.J.; WARWICK, S.I.; JOHNSON, E.N.; HRYNEWICH, B.; LOZINSKI, C. Growth characterization of kochia (Kochia scoparia) with substitutions at $\mathrm{Pro}_{197}$ or $\operatorname{Trp}_{574}$ conferring resistance to acetolactate synthase-inhibiting herbicides. Weed Science, v.61, p.267-276, 2013. DOI: 10.1614/WS-D-12-00116.1.

LI, M.; YU, Q.; HAN, H.; VILA-AIUB, M.; POWLES, S.B. ALS herbicide resistance mutations in Raphanus raphanistrum: evaluation of pleiotropic effects on vegetative growth and ALS activity. Pest Management Science, v.69, p.689-695, 2013. DOI: $10.1002 /$ ps.3419.

MAGALHÃES, A.C.N. Análise quantitativa do crescimento. In: FERRI, M.G. (Coord.). Fisiologia vegetal. São Paulo: EPU/ EDUSP, 1979. v.1, p.331-350.

OERKE, E.-C. Crop losses to pests. Journal of Agricultural Science, v.144, p.31-43, 2006. DOI: 10.1017/S0021859605005708.

PASSINI, T.; CHRISTOFFOLETI, P.J.; YADA, I.F.U. Competitivity of the common-bean plant relative to the weed alexandergrass [Brachiaria plantaginea (Link) Hitch.]. Scientia Agricola, v.60, p.259-268, 2003. DOI: 10.1590/S0103-90162003000200009.

POWLES, S.B.; YU, Q. Evolution in action: plants resistant to herbicides. Annual Review of Plant Biology, v.61, p.317-347, 2010.

RADOSEVICH, S.R.; HOLT, J.S.; GHERSA, C.M. Ecology of weeds and invasive plants: relationship to agriculture and natural resource management. $3^{\text {rd }}$ ed. Hoboken: John Wiley \& Sons, 2007. 454p. DOI: $10.1002 / 9780470168943$.

RIGOLI, R.P.; AGOSTINETTO, D.; SCHAEDLER, C.E.; DAL MAGRO, T.; TIRONI, S. Habilidade competitiva relativa do trigo (Triticum aestivum) em convivência com azevém (Lolium multiflorum) ou nabo (Raphanus raphanistrum). Planta Daninha, v.26, p.93-100, 2008. DOI: 10.1590/S0100-83582008000100010. 
SANTOS, H.G. dos; JACOMINE, P.K.T.; ANJOS, L.H.C. dos; OLIVEIRA, V.A. de; LUMBRERAS, J.F.; COELHO, M.R.; ALMEIDA, J.A. de; CUNHA, T.J.F.; OLIVEIRA, J.B. de. Sistema brasileiro de classificação de solos. 3.ed. rev. e ampl. Brasília: Embrapa, 2013. 353p.

SCHAEDLER, C.E.; NOLDIN, J.A.; AGOSTINETTO, D.; DAL MAGRO, T.; FONTANA, L.C. Germination and growth of Fimbristylis miliacea biotypes resistant and susceptible to acetolactate synthase-inhibiting herbicides. Planta Daninha, v.31, p.687-694, 2013. DOI: 10.1590/S0100-83582013000300020.

SIBONY, M.; RUBIN, B. The ecological fitness of ALS-resistant Amaranthus retroflexus and multiple-resistant Amaranthus blitoides. Weed Research, v.43, p.40-47, 2003. DOI: 10.1046/j.13 65-3180.2003.00315.x.

TARDIF, F.J.; RAJCAN, I.; COSTEA, M. A mutation in the herbicide target site acetohydroxyacid synthase produces morphological and structural alterations and reduces fitness in Amaranthus powellii. New Phytologist, v.169, p.251-264, 2006. DOI: 10.1111/j.1469-8137.2005.01596.x.

URCHEI, M.A.; RODRIGUES, J.D.; STONE, L.F. Análise de crescimento de duas cultivares de feijoeiro sob irrigação, em plantio direto e preparo convencional. Pesquisa Agropecuária Brasileira, v.35, p.497-506, 2000. DOI: 10.1590/ S0100-204X2000000300004.

VARGAS, L.; ROMAN, E.S.; RIZZARDI, M.A.; SILVA, V.C. Alteração das características biológicas dos biótipos de azevém (Lolium multiflorum) ocasionada pela resistência ao herbicida glyphosate. Planta Daninha, v.23, p.153-160, 2005. DOI: 10.1590/ S0100-83582005000100018.

VILA-AIUB, M.M.; NEVE, P.; POWLES, S.B. Evidence for an ecological cost of enhanced herbicide metabolism in Lolium rigidum. Journal of Ecology, v.97, p.772-780, 2009a. DOI: 10.1111/j.1365-2745.2009.01511.x.

VILA-AIUB, M.M.; NEVE, P.; POWLES, S.B. Fitness costs associated with evolved herbicide resistance alleles in plants. New Phytologist, v.184, p.751-767, 2009b. DOI: 10.1111/j.1469-8137. 2009.03055.x.

YU, Q.; HAN, H.; VILA-AIUB, M.M.; POWLES, S.B. AHAS herbicide resistance endowing mutations: effect on AHAS functionality and plant growth. Journal of Experimental Botany, v.61, p.3925-3934, 2010. DOI: 10.1093/jxb/erq205.

Recebido em 29 de novembro de 2014 e aprovado em 3 de maio de 2016 